\title{
Clinical, Biochemical and Echocardiographic Characteristics of Severe SARS-COV-2 Infection-Correlates of In-hospital Morbidity and Mortality
}

\author{
Sabha Bhatti ${ }^{1}$, Justin Johanessen ${ }^{1}$, Ikenna Erinne ${ }^{1}$, Kulin Shah ${ }^{1}$, Ayesha Salahuddin ${ }^{1}$, and \\ Abdul Hakeem ${ }^{1}$ \\ ${ }^{1}$ Rutgers Robert Wood Johnson Medical School New Brunswick
}

July 28, 2020

\begin{abstract}
Background: Patients with cardiovascular disease are more susceptible to coronavirus disease 2019 (COVID-19) and have worse outcomes when infected. This study reports the largest and most comprehensive echocardiographic evaluation of patients with severe COVID-19 at a quaternary care hospital in the second most affected state in the US, New Jersey. Methods: Clinical, biochemical and echocardiographic features of consecutive patients with severe COVID-19 undergoing echocardiography were studied. Clinical outcome data including length of stay, requirement of mechanical ventilation and in-hospital mortality were collected. Results: 987 patients with confirmed COVID-19 infection were treated at our institution of which 146 consecutive patients (15\%) underwent echocardiographic evaluation. Median age was 63 years $; 37 \%$ were females, $21 \%$ had known CAD and $20 \%$ had CKD. $57 \%$ of patients required mechanical ventilation and $50 \%$ required vasopressors . $31 \%$ of patients died during the index hospitalization. There was a high prevalence of echocardiographic abnormalities including right ventricular dilation (33\%) or dysfunction (21\%), left ventricular dysfunction (20\%), and pericardial effusion (13\%). Multiple biomarkers including troponin $\mathrm{T}$, pro BNP, dimer and CRP were strongly associated with echocardiographic abnormalities and in-hospital mortality. On Cox regression analysis, age (HR 1.04/year) and CAD (HR 2.4) were independent predictors of mortality. Conclusions: Severe COVID-19 infection is accompanied by a significant burden of echocardiographic abnormalities that are strongly correlated with higher degrees of inflammation and biomarker elevation. Additional investigation is warranted in assessing the role of a biomarker-guided approach for early cardiac surveillance using echocardiography in further risk stratifying patients and tailoring adjunctive therapy.
\end{abstract}

\section{Introduction:}

COVID-19, caused by SARS-CoV-2, is a global pandemic that is evolving rapidly. The incidence, prevalence and potential mechanisms of cardiovascular manifestations in COVID 19 patients remain an area of active investigation. Patients with cardiovascular disease are more susceptible to COVID-19 and have a more severe clinical course once infected (1). However, it is not known if the presence of cardiovascular disease poses independent risk or whether higher rates of observed adverse outcomes are confounded by other concomitant factors such as increased age or comorbid conditions. Multiple studies, mostly emanating from China, have demonstrated myocardial injury with cardiac biomarker elevation in 7-28\% of patients and an association with increased risk of mortality (2). COVID-19 infection has also been associated with other cardiovascular abnormalities including myocarditis, cardiac arrhythmias and cardiomyopathy (3). Right-sided heart failure as a result of massive pulmonary embolism (PE) due to COVID-19 has been documented in several recent reports $(4,5)$. Right ventricular $(\mathrm{RV})$ dysfunction has also recently been shown to be a predictor of mortality $(6)$. 
Given the risk of healthcare worker and patient exposure and potential contamination of equipment, echocardiographic evaluation is not frequently performed in these patients despite biochemical evidence of myocardial injury. As such, while there are multiple small individual reports of cardiac involvement in COVID-19, there has been no comprehensive evaluation of the potential clinical, biochemical and echocardiographic correlates in COVID-19 patients thus far.

As a quaternary care hospital in the state of New Jersey, one of the epicenters of COVID-19 infection in the U.S, we were able to gain unique insight into the clinical course, cardiovascular manifestations, and outcomes of patients with severe COVID-19. The purpose of this study was to evaluate the prevalence of particular cardiovascular abnormalities identified on transthoracic echocardiography, their association with baseline cardiovascular risk factors and the potential role of clinical and laboratory features as predictors of mortality in patients with severe COVID-19.

\section{Methods:}

\section{Study population:}

This observational study was performed at Rutgers Robert Wood Johnson University Hospital in NJ, USA. 146 consecutive adult patients with severe COVID-19 infection who underwent transthoracic echocardiography from March 17, 2020 to May 12, 2020 were studied. (Figure 1 a) The study was approved by the Rutgers Robert Wood Johnson Medical School institutional review board.

\section{Clinical Data:}

Patient demographics, medical history, laboratory examinations, comorbidities, treatments and outcomes were retrieved from electronic medical records. Cardiac biomarkers measured on admission, closest to the time of echocardiography and peak values included troponin T, pro B-type natriuretic peptide (pro BNP), ferritin, D-dimer, C reactive protein (CRP) and triglyceride levels were recorded. The data was independently reviewed and entered into a database. The final follow-up date was May 17, 2020.

\section{Transthoracic Echocardiography}

Bedside transthoracic echocardiographic examinations were performed in all patients with the EPIQ ultrasound systems (Philips Medical Systems, Andover, MA, USA). Limited 2D and Doppler echocardiography were performed based on the laboratory's pandemic policy. Standard views were acquired to assess LV and RV function, valves (if indicated) and pericardial effusion. Left ventricular (LV) end-systolic volume (ESV) and end-diastolic volume (EDV), and ejection fraction (EF) were measured by biplane Simpson's method. RV size was determined from the apical 4-chamber view. Opacifying agents were used if more than 2 consecutive wall segments were not well visualized. Acquired Images were analyzed by two independent observers (SB and AS) blinded to clinical and outcomes data.

\section{Statistical analysis}

Continuous numeric variables were expressed as mean \pm SD or medians (IQR), and compared with a twosample t test and one-way analysis of variance (for normally distributed data), or Mann-Whitney test and Kruskal-Wallis test (for non-normal distribution of data). Categorical variables were expressed as frequency number (\%), and compared using $\chi 2$ test or Fisher's exact test. Survival curves were obtained by the Kaplan-Meier analysis and compared using log-rank test. Receiver operator characteristics (ROC) analysis was undertaken to compute the best predictive threshold of significant biomarker elevation for mortality prediction. Estimations of mortality predictors were performed using univariate and multivariate Cox regression models. All statistical calculations were performed using MedCalc Statistical Software version 19.2 (MedCalc Software, Ostend, Belgium; http://www.medcalc.org; 2020).

\section{Results:}

\section{Clinical Characteristics}


Clinical characteristics of patients with COVID-19 are shown in Table 1. The mean age of COVID-19 patients was 63 years, $90(63 \%)$ patients were men. 36\% of patients were Hispanic, $29 \%$ Caucasian, $18 \%$ Asian and $16 \%$ African American. Hypertension was present in $66 \%$, coronary artery disease (CAD) in $21 \%$, diabetes in $46 \%$, and chronic kidney disease (CKD) in $20 \%$ of patients. $57 \%$ required mechanical ventilation at some point in their hospitalization and half of all patients required vasopressor support. Body mass index (BMI) was similar in both groups.

Patients who died were older, had history of CAD ( $42 \%$ versus $12 \%)$, CKD (31\% versus $15 \%$ ), and were more likely to be smokers (11\% versus $3 \%$ ). They were also more likely to be febrile with temperatures greater than 101 degrees Fahrenheit, mechanically ventilated or on vasopressors. (Table 1)

\section{In-hospital Medications}

Table 2 shows the different medications utilized during the study period, many of which were part of clinical trials, at our institution. Almost $60 \%$ of patients were treated with systemic steroids and $73 \%$ were receiving therapeutic anticoagulation. Hydroxychloroquine, tocilizumab and remdesivir were used in $61 \%, 39 \%$ and $9 \%$ of patients respectively. There were no mortality differences in the use of COVID-19-specific treatments, however there was a significantly higher use of vasopressor medications in patients who died $(73 \%$ vs $40 \% ; p=$ 0.0002). $29 \%$ of all patients were on angiotensin converting enzyme inhibitors/angiotensin receptor blockers $(\mathrm{ACEi} / \mathrm{ARB})$ and while not statistically significant, there was a trend towards more patients on ACEi/ARB and increased mortality ( $40 \%$ versus $24 \% ; p=0.06)$.

\section{Biomarkers}

Patients who died had significantly higher Creatinine, peak Pro-BNP, RP, D-dimer, ferritin and triglyceride levels compared to those who survived as shown in Table 3a. Table $3 \mathrm{~b}$ demonstrates the predictive threshold of 6 biomarkers (CRP, D-Dimer, Ferritin, Pro-BNP, Troponin T, and Triglyceride) for mortality prediction as computed on ROC analysis.

\section{Echocardiographic Findings}

Indications for echocardiography are demonstrated in figure $1 \mathrm{~b}$. Median time between admission and echocardiography was 5 days (IQR1-10) (Figure 1c). Most common indications for echocardiography were evaluation for cardiac size and function. LV function was categorized as less than or greater than $45 \%$. RV function was classified as normal versus dilated and function was classified as normal versus reduced. The overall LV EF was preserved at $55 \%$ and $20 \% \%$ patients who had LVEF $<45 \%$. RV dilation and dysfunction were seen in $33 \%$ and $21 \%$ patients respectively. A dilated or dysfunctional RV was found in $39.4 \%$ patients. Pericardial effusion was found in $13 \%$ of patients. (Table 4 )

Patients with RV dilation/dysfunction had significantly higher peak troponin T of 0.05 (IQR 0.01-0.16) compared to those without (peak trop T 0.01 (IQR 0.00-0.05); $\mathrm{p}<0.0001$. They also had higher levels of pro BNP (4618 vs. 2092), CRP (26.2 vs. 22), ferritin (1577 vs. 1170) and D-Dimer (12553 vs 5570) compared to those without RV dilation or dysfunction. Patients with LV dysfunction $(\mathrm{EF}<45 \%)$ had higher levels of d-dimer, pro BNP and troponin T compared to those without LV dysfunction.

\section{Predictors of Outcomes}

Median length of stay was 13 days (IQR 7.7-24). 45 patients died (31\%), 71 were discharged (49\%) and $30(20 \%)$ patients were still hospitalized at the time of most recent follow up. Figure 2a shows KM survival analysis for patient stratified based on age $>60$ and $<60$ years with a significantly higher mortality $(40 \%)$ for patients more than 60 years old (compared to $14 \%$ for those 60 and below). Patients with preexisting CAD had a significantly higher mortality (62\%) compared to those without (22\%) Log Rank P value $<0.001$ (Figure $2 \mathrm{~b}$ ). Use of troponin $\mathrm{T}$ as a predictive instrument yielded excellent discrimination in survival such that patients with troponin $\mathrm{T}>0.01$ had $43 \%$ mortality compared to $14 \%$ for those with troponin $\mathrm{T}<0.01$. (Figure 3a). While the median EF was within normal limits, patients with LV dysfunction $<45 \%$ had numerically higher mortality compared to those with $\mathrm{EF}>45 \%$ ( $37 \%$ vs $27 \%$; Log Rank $\mathrm{P}=0.16$ ) (Figure 
3b). On multivariate Cox analysis, age and history of CAD were independent predictors of mortality. (Table $5)$.

\section{Discussion:}

To our knowledge, this is the largest study to evaluate the cardiovascular prevalence, biomarkers, and echocardiographic features in patients with severe COVID-19 infection. The main findings of the study can be summarized as follows: a large proportion of patients with severe COVID-19 infection have echocardiographic abnormalities including right and left ventricular dysfunction. The presence of these abnormalities is strongly associated with underlying clinical and biochemical severity of COVID-19 illness. Several of these clinical and biochemical variables are powerful predictors of mortality and can be used as effective tools to refine risk stratification and filters for further clinical testing including echocardiography.

Coronavirus disease 2019 (COVID-19), the novel infection caused by the severe acute respiratory syndrome coronavirus-2 (SARS-CoV-2), was first detected in China in December, 2019 and declared a global pandemic by the World Health Organization in early 2020 (7). It has posed a significant health care threat in the United States where, as of May 22, 2020, over 1.5 million people have been infected resulting in over 90,000 deaths (8). Early reports from China demonstrated that a significant majority of patients had mild symptoms (no pneumonia or mild pneumonia) but patients with co-morbid conditions, such as cardiovascular disease and cardiovascular risk factors (diabetes and hypertension), have more severe clinical course and higher case fatality rates (9). Severe pneumonia resulting in acute respiratory distress syndrome (ARDS) and acute respiratory failure remain the main causes of mortality in critically ill patients with COVID19 (10). Furthermore, recent studies have identified the interplay of coagulation activation and sustained inflammatory response as an important factor contributing to increased complications and poorer outcomes in COVID-19 patients. These include the development of ARDS, multi organ failure including acute kidney injury, disseminated intravascular coagulation, clinically significant pulmonary embolism (PE), deep vein thrombosis (DVT) and an exaggerated inflammatory activation with hypercytokinaemia $(3,4,7)$.

The Burden of Right ventricular dysfunction in Critically Ill COVID-19 illness

Numerous reports have confirmed a hypercoagulable state associated with severity of COVID-19 infection with multiple manifestations including a significantly increased risk of deep venous thrombosis and pulmonary embolism. While respiratory compromise in coronavirus was initially mostly attributed to ARDS, the contribution of embolic phenomena is now more appreciated. In our cohort of COVID 19 patients, while the median D-dimer level was significantly elevated at 7283 for the entire cohort, this was twice as high for patients who died. Previous work has demonstrated the RV involvement that occurs as a result of this increased right sided afterload. (11) This was confirmed in our cohort with $32 \%$ of patients experiencing RV dilatation and 21\% experiencing reduction in RV systolic function. However, the diagnosis of pulmonary embolism in COVID 19 patients is difficult. First, many of them experience acute kidney injury as a result of their infection that is thought to be multifactorial and related to micro thrombi, endothelial dysfunction, volume status, etc. (2). This acute kidney injury precludes many patients from receiving diagnostic CT pulmonary angiograms. Second, many radiology departments attempt to limit the scanning of COVID 19 patients due to extensive decontamination required after each study. This highlights the additional role of bedside cardiac ultrasound in the management of these patients. At our institution, many patients with ongoing hypoxia received echocardiograms and were presumptively treated for PE with TPA if evidence of RV strain was found.

In a recently published report of 110 patients from New York City, undergoing echocardiographic evaluation the prevalence of RV dilation was 31\% (out of 110 patients; 30\% intubated and in-hospital mortality 20\%).(11) Our findings are similar with $32 \%$ patients having evidence of RV dilation. Another recent publication from China demonstrated the added value of RV strain in predicting mortality in 120 COVID-patients (12.5\% intubated and $15 \%$ in hospital mortality) (6). While strain analysis is a more sensitive marker of RV dysfunction, we were not able to assess that given significant differences between our cohort and Li et al's report. Only $12.5 \%$ of their cohort was mechanically ventilated compared to $57 \%$ in our study. Furthermore, 
the median BMI in our cohort was 30 compared to 23 in Li et al study. This large percentage of intubated and obese patients limited the quality necessary for reliable strain imaging. Our study did demonstrate however that biomarkers could predict the presence of RV dilation/dysfunction. Patients with signs of RV involvement had significant higher pro BNP (4618 vs 2092) and D dimer (12553 vs 5570) compared to those normal right ventricles. This RV strain is presumably a manifestation of subclinical pulmonary embolism, hypoxemic vasoconstriction and other sequela of infection. While future studies are needed, this would seem to suggest that there exists a biomarker threshold over which a cardiac ultrasound should be performed to assess for RV dysfunction.

Biochemical Myocardial Injury, echocardiographic abnormalities and outcomes

Our results demonstrate that cardiac biomarkers were a significant predictor of mortality for those with COVID-19. Patients with negative troponins (peak troponin less than or equal to 0.01) had a mortality rate of $16 \%$ compared to $43 \%$ with any elevation ( $\mathrm{p}<.003)$. Pro BNP was also implicated, with levels over 19280 serving as an independent risk factor for mortality $(\mathrm{p}<.002)$. In our cohort $20 \%$ of the patients had LVSD or RVD, which correlated with the worsening biochemical profile. There was numerically higher mortality in the patients with left ventricle dysfunction but it did not reach statistical significance. One possible explanation for this finding could be the timing between echocardiography and time of death, which was a median of 5 days. It is likely that the cardiac function continued to worsen as these patients got sicker with worsening biochemical profiles and increasing pressor requirement.

Pre-existing CAD and risk of mortality

One of the most striking and statistically significant findings of our cohort was the association between preexisting $\mathrm{CAD}$ and mortality. Patients with $\mathrm{CAD}$ had $62 \%$ mortality compared to $22 \%$ for those without prior CAD ((HR 2.4; 95\% CI 1.16-4.9; $P=0.01)$ (figure 5). The association between viral illness and cardiovascular morbidity and mortality is well known. Previous work has shown much higher rates of influenza related death among those patients with cardiovascular disease. (13) Systemic illness puts significant stress on the heart as it tries to meet increased metabolic demands. In our cohort, we found several important differences between patients with preexisting CAD and those without that could explain the high mortality seen in these patients. Patients with preexistent CAD were significantly older (77 vs 61 years), had a higher prevalence of CKD (42 vs 14\%), underlying CHF (26\% vs $6 \%$ ), EF $<45 \%$ ( $42 \%$ vs $14 \%$ ) and higher levels of pro BNP and peak troponin T levels compared to those without CAD (supplemental Table). Interestingly, the level of inflammatory markers and D- dimer were not significantly different. While the exact mechanism for worse outcomes in COVID-19 patients with preexisting CAD is unclear, several theories have been suggested. Severe hypoxemia and cytokine storm, mediated by an imbalanced response among subtypes of $\mathrm{T}$ helper cells, may contribute to myocardial injury in COVID-19 patients and pose a greater risk in patients with a history of CAD and heart failure $(7,9)$. SARS-CoV has also been shown to directly cause myocardial inflammation via interaction with angiotensin-converting enzyme 2, a known receptor for SARS-COV-2 expressed on myocytes and vascular endothelial cells. $(14,15)$

\section{Limitations}

This is a relatively small, single-center study on consecutive patients hospitalized with severe COVID-19. This limits the generalizability of the findings discussed. Echocardiograms were ordered at the discretion of treating physicians and represent roughly $15 \%$ of patients treated at our center, most of whom already had unfavorable clinical course at the time of the study. This accounts in part for the higher mortality (31\%) seen in our cohort compared to the previous Wuhan echocardiogram report (6) which reported a $15 \%$ mortality in their imaged patients. Given ongoing efforts to limit the spread of COVID-19, routine echocardiograms that are unlikely to change patient management were minimized. When performed, echocardiographic studies were limited to features deemed most clinically relevant: LV size and function, RV size and function, significant valvular abnormalities, and presence of pericardial effusions. Measurements of ventricular strain and LV diastolic function were not obtained. Nonetheless, this experience represents the largest and most comprehensive report on echocardiogram features and biochemical cardiac markers in COVID 19 patients 
thus far.

\section{Clinical Implications \& Conclusions}

Severe COVID-19 infection is accompanied by a significant burden of echocardiographic abnormalities that are strongly correlated with the degree of inflammation and biomarker elevation. Such patients form a very high risk group. Further study is warranted to understand the role of early cardiac surveillance using echocardiography in risk stratifying patients and the use of adjunct therapy tailored according to these findings.

\section{References}

1) Inciardi RM, Adamo M, Lupi L, et al. Characteristics and Outcomes of Patients Hospitalized for COVID19 and Cardiac Disease in Northern Italy. Eur Heart J. 2020;41(19):1821-1829.

2) Cheng R, Leedy D. COVID-19 and acute myocardial injury: the heart of the matter or an innocent bystander? Heart. 2020:heartjnl-2020-317025. doi: 10.1136/heartjnl-2020-317025.

3) Driggin E, Madhavan MV, Bikdeli B, et al. Cardiovascular Considerations for Patients, Health Care Workers, and Health Systems During the COVID-19 Pandemic. J Am Coll Cardiol. 2020;75(18):2352-2371.

4) Marginean A, Masic D, Brailovsky Y, Fareed J, Darki A. Difficulties of Managing Submassive and Massive Pulmonary Embolism in the era of COVID-19. JACC Case Rep. (2020). doi: https://doi.org/10.1016/j.jaccas.2020.05.017

5) Ullah W, Saeed R, Sarwar U, et al. COVID-19 complicated by Acute Pulmonary Embolism and RightSided Heart Failure [published online ahead of print, 2020 Apr 17]. JACC Case Rep.

6) Li Y, Li H, Zhu S, Xie Y, Wang B, He L, Zhang D, Zhang Y, Yuan H, Wu C, Sun W, Zhang Y, Li M, Cui L, Cai Y, Wang J, Yang Y, Lv Q, Zhang L, Xie M. Prognostic Value of Right Ventricular Longitudinal Strain in Patients with COVID-19,..JACC Cardiovas Imaging (2020), doi: https://doi.org/10.1016/j.jcmg.2020.04.014.

7) Inciardi RM, Adamo M, Lupi L, et al. Characteristics and outcomes of patients hospitalized for COVID-19 and cardiac disease in Northern Italy. Eur Heart J. 2020;41(19):1821-1829

8) https://www.cdc.gov/coronavirus/2019-ncov/cases-updates/cases-in-us.html. Accessed May 23, 2020

9) Clerkin KJ, Fried JA, Raikhelkar J, et al. COVID-19 and Cardiovascular Disease. Circulation. 2020;141(20):1648-1655.

10) Shi S, Qin M, Cai Y, et al. Characteristics and Clinical Significance of Myocardial Injury in Patients with Severe Coronavirus Disease 2019 [published online ahead of print, 2020 May 11]. Eur Heart J. 2020;ehaa408. doi:10.1093/eurheartj/ehaa408

11) Argulian E, Sud K, Vogel B et al . Right Ventricular Dilation in Hospitalized Patients with COVID-19 Infection. JACC Cardiovascular Imaging (2020), doi: https://doi.org/10.1016/j.jcmg.2020.05.010.

12) Ronco C, Reis T, Husain-Syed F. Management of Acute Kidney Injury in Patients with COVID 19. The Lancet: Respiratory Medicine. May 142020.

13) Nguyen J, Wan Y, Ito K, Matte T, Shama J, Kinney P. Seasonal Influenza Infections and Cardiovascular Mortality. JAMA Cardiology. 2016;1(3):274-281. doi:10.1001/jamacardio.2016.0433

14) Zhou F, Yu T, Du R, et al. Clinical course and Risk Factors for Mortality of Adult Inpatients with COVID-19 in Wuhan, China: A Retrospective Cohort Study. Lancet. 2020;395(10229):1054-1062. doi:10.1016/S0140-6736(20)30566-3

15) Huang L, Zhao P, Tang D, et al. Cardiac Involvement in Recovered COVID-19 Patients Identified by Magnetic Resonance Imaging. JACC Cardiovas Imaging (2020), doi: https://doi.org/10.1016/j.jcmg.2020.05.004.

Table 1: Baseline characteristics 


\begin{tabular}{lllll}
\hline & All $(\mathbf{N = 1 4 6})$ & Alive (N=101) & Dead (N=45) & P Value \\
\hline Age & $63(52-72)$ & $60+/-15$ & $68+/-14$ & 0.002 \\
Males & $90(63 \%)$ & $60(59 \%)$ & $30(71 \%)$ & 0.4 \\
Race & & & & NS \\
Hispanic & $52(36 \%)$ & $39(38 \%)$ & $13(29 \%)$ & \\
African American & $24(16 \%)$ & $16(16 \%)$ & $8(18 \%)$ & \\
Caucasian & $43(29 \%)$ & $27(26 \%)$ & $16(36 \%)$ & \\
Asian & $27(18 \%)$ & $19(20 \%)$ & $8(18 \%)$ & \\
Diabetes & $68(46 \%)$ & $47(46 \%)$ & $22(49 \%)$ & 0.79 \\
Hypertension & $96(66 \%)$ & $64(63 \%)$ & $32(71 \%)$ & 0.11 \\
Dyslipidemia & $59(40 \%)$ & $38(37 \%)$ & $21(47 \%)$ & 0.14 \\
CAD & $31(21 \%)$ & $12(12 \%)$ & $19(42 \%)$ & $<0.0001$ \\
CHF & $15(10 \%)$ & $9(9 \%)$ & $6(13 \%)$ & 0.41 \\
COPD & $15(10 \%)$ & $11(11 \%)$ & $4(9 \%)$ & 0.71 \\
Atrial fibrillation & $17(12 \%)$ & $13(13 \%)$ & $4(9 \%)$ & 0.49 \\
CKD & $29(20 \%)$ & $15(15 \%)$ & $14(31 \%)$ & 0.02 \\
Smoking & $8(5 \%)$ & $3(3 \%)$ & $5(11 \%)$ & 0.04 \\
Fevers $>$ 101 F & $91(62 \%)$ & $58(57 \%)$ & $33(73 \%)$ & 0.06 \\
Mechanical Ventilation & $83(57 \%)$ & $49(48 \%)$ & $34(75 \%)$ & 0.002 \\
Pressors & $73(50 \%)$ & $40(40 \%)$ & $33(73 \%)$ & 0.0002 \\
\hline
\end{tabular}

${ }^{*} \mathrm{CAD}=$ coronary artery disease $\mathrm{CHF}=$ congestive heart failure; $\mathrm{COPD}=$ chronic obstructive pulmonary disease; $\mathrm{CKD}=$ chronic kidney disease

Table 2- In hospital Medications

\begin{tabular}{lllll}
\hline Medication & Overall & Alive & Dead & P-value \\
\hline Hydroxychloroquine & $89(61 \%)$ & $59(58 \%)$ & $30(67 \%)$ & 0.34 \\
Azithromycin & $31(21 \%)$ & $23(23 \%)$ & $8(18 \%)$ & 0.49 \\
Tocilizumab & $57(39 \%)$ & $38(38 \%)$ & $19(42 \%)$ & 0.6 \\
Remdesivir & $13(9 \%)$ & $9(9 \%)$ & $4(9 \%)$ & 0.99 \\
Therapeutic AC & $107(73 \%)$ & $75(74 \%)$ & $32(71 \%)$ & 0.69 \\
Steroid & $86(59 \%)$ & $56(55 \%)$ & $30(66 \%)$ & 0.20 \\
ACE -/ARB & $43(29 \%)$ & $25(24 \%)$ & $18(40 \%)$ & 0.06 \\
Pressors & $73(50 \%)$ & $40(40 \%)$ & $33(73 \%)$ & 0.0002 \\
QTc(milliseconds) & $450(430-483)$ & $446(429-474)$ & $463(435-495)$ & 0.13 \\
\hline
\end{tabular}

$\mathrm{ACE}=$ angiotensin converting enzyme; $\mathrm{ARB}=$ angiotensin receptor blockers

Table 3 a- Biochemical Profile

\begin{tabular}{llllll}
\hline Variable & Normal Range & $\begin{array}{l}\text { Overall } \\
\text { Median(IQR) }\end{array}$ & Alive & Dead & P-value \\
\hline $\begin{array}{l}\text { Creatinine } \\
\text { (mg/dL) }\end{array}$ & $0.5-1.2$ & $1.1(0.8-1.9)$ & $0.90(0.77-1.5)$ & $1.3(1-2.3)$ & 0.007 \\
$\begin{array}{l}\text { Peak Pro BNP } \\
(\mathbf{p g} / \mathbf{m l})\end{array}$ & $<300$ & $2742(846-16744)$ & $1886(470-5974)$ & $6087(1441-$ & 0.003 \\
$\begin{array}{l}\text { Peak Trop T } \\
(\text { ng/mL) }\end{array}$ & $<0.01$ & $0.01(0.00-0.06)$ & $0.01(0.00-0.06)$ & $0.05(0.02-0.36)$ & 0.0007
\end{tabular}




\begin{tabular}{llllll}
\hline Variable & Normal Range & $\begin{array}{l}\text { Overall } \\
\text { Median(IQR) }\end{array}$ & Alive & Dead & P-value \\
\hline $\begin{array}{l}\text { Peak CRP } \\
(\mathbf{m g} / \text { dL) }\end{array}$ & $0-0.7$ & $25(13-33)$ & $20.9(11.4-31)$ & $32(23.5-38.6)$ & 0.0005 \\
$\begin{array}{l}\text { Peak D-dimer } \\
(\mathbf{n g} / \mathbf{m L})\end{array}$ & $0-500$ & $7283(2911-$ & $6397(1881-$ & $14487(4461-$ & 0.01 \\
$\begin{array}{l}\text { Peak Ferritin } \\
(\mathbf{n g} / \mathbf{m L})\end{array}$ & $20-335$ & $26754)$ & $21228)$ & $95442)$ & \\
$\begin{array}{l}\text { Peak } \\
\begin{array}{l}\text { Triglyceride } \\
(\mathbf{m g} / \mathbf{d L})\end{array}\end{array}$ & $40-150$ & $1337(637-2719)$ & $1191(575-2457)$ & $1876(1028-2847)$ & 0.005 \\
\hline
\end{tabular}

$\mathrm{BNP}=$ brain natriuretic peptide; $\mathrm{CRP}=\mathrm{C}$ reactive protein

Table 3b- Predictive threshold of biochemical markers for mortality prediction

\begin{tabular}{llll}
\hline Peak Value & Threshold value & AUC & P value \\
\hline CRP & $>22.76$ & 0.67 & 0.004 \\
D-dimer & $>82403$ & 0.63 & 0.009 \\
Ferritin & $>994$ & 0.60 & 0.04 \\
Pro BNP & $>19280$ & 0.68 & 0.002 \\
Troponin T & $>0.01$ & 0.68 & 0.003 \\
Triglyceride & $>122$ & 0.66 & 0.007 \\
\hline
\end{tabular}

$\mathrm{AUC}=$ area under curve; $\mathrm{BNP}=$ brain natriuretic peptide; $\mathrm{CRP}=\mathrm{C}$ reactive protein

Table 4- Echocardiographic findings

\begin{tabular}{lllll}
\hline & Overall & Alive & Dead & P value \\
\hline LVEF(\%) & $55(55-60)$ & $55(55-60)$ & $55(45-60)$ & 0.66 \\
LVEF $<45 \%$ & $30(20 \%)$ & $19(18 \%)$ & $11(24 \%)$ & 0.18 \\
Dilated RV & $48(33 \%)$ & $34(33 \%)$ & $14(31 \%)$ & 0.79 \\
RV dysfunction & $31(21 \%)$ & $21(20 \%)$ & $10(22 \%)$ & 0.71 \\
Pericardial effusion & $19(13 \%)$ & $13(13 \%)$ & $6(13 \%)$ & 0.90 \\
\hline
\end{tabular}

$\mathrm{LVEF}=$ left ventricular ejection fraction; $\mathrm{RV}=$ right ventricle

Table 5: Multivariate Model: Clinical Predictors of Mortality

\begin{tabular}{lll}
\hline & $\operatorname{HR}(95 \% \mathrm{CI})$ & $\mathrm{P}$ \\
\hline Age & $1.04(1.01-1.07)$ & 0.002 \\
Gender & $0.61(0.30-1.2)$ & 0.18 \\
CAD & $2.40(1.16-4.9)$ & 0.01 \\
CKD & $1.20(0.57-2.5)$ & 0.61 \\
Smoking & $0.97(0.35-2.6)$ & 0.95 \\
Fevers $>101$ & $1.19(0.54-2.6)$ & 0.66 \\
Mechanical Ventilation & $2.10(0.92-4.83)$ & 0.07 \\
\hline
\end{tabular}


$\mathrm{HR}=$ hazards ratio $\mathrm{CAD}=$ coronary artery disease; $\mathrm{SKD}=$ chronic kidney disease Supplementary Table

\begin{tabular}{|c|c|c|c|}
\hline & $\begin{array}{l}\text { Pre Existing CAD } \\
(\mathrm{N}=31)\end{array}$ & No CAD $(\mathrm{N}=114)$ & $P$ value \\
\hline Age & $77(66-82)$ & $61(51-67)$ & $<0.0001$ \\
\hline CKD & $13(42 \%)$ & $16(14 \%)$ & 0.0006 \\
\hline CHF & $8(26 \%)$ & $7(6 \%)$ & 0.001 \\
\hline Fevers $>101$ & $16(52 \%)$ & $74(65 \%)$ & 0.17 \\
\hline Intubation & $16(52 \%)$ & $66(58 \%)$ & 0.53 \\
\hline Creatinine & $1.5(1.1-2.2)$ & $1(0.8-1.5)$ & 0.0028 \\
\hline QTc & $462(437-499)$ & $446(429-479)$ & 0.1 \\
\hline $\mathrm{EF}<45 \%$ & $13(42 \%)$ & $16(14 \%)$ & 0.0006 \\
\hline RVD & $13(43 \%)$ & $40(36 \%)$ & 0.54 \\
\hline Peak CRP & $23.7(12.6-31.2)$ & $25.9(13-24)$ & 0.47 \\
\hline Peak D-dimer & $6871(3662-41304)$ & $7343(2215-23361)$ & 0.364 \\
\hline Peak Ferritin & $1303(600-2866)$ & $1360(637-2543)$ & 0.82 \\
\hline Peak pro BNP & $5626(1358-28718)$ & $2742(592-11276)$ & 0.05 \\
\hline Peak Trop T & $0.05(0.02-0.15)$ & $0.01(0.00-0.07)$ & 0.001 \\
\hline
\end{tabular}

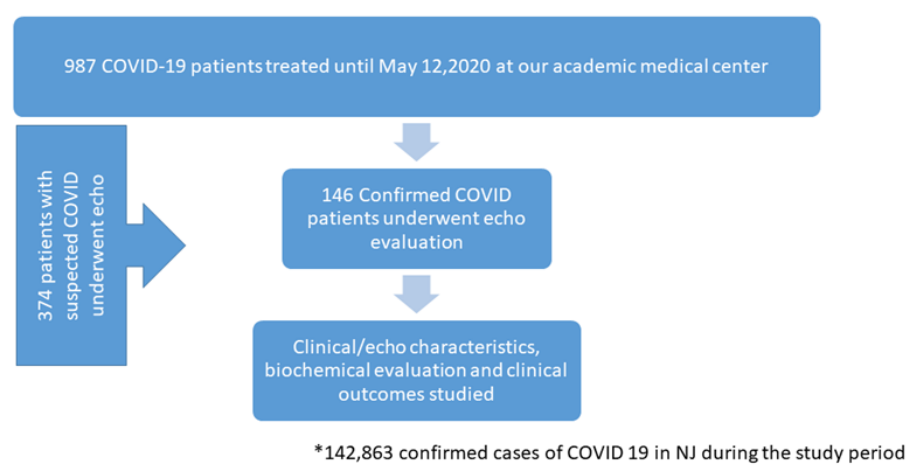

Figure 1 a- Flow Chart of Study population

Figure 1b- 
INDICATIONS FOR ECHOCARDIOGRAPHY

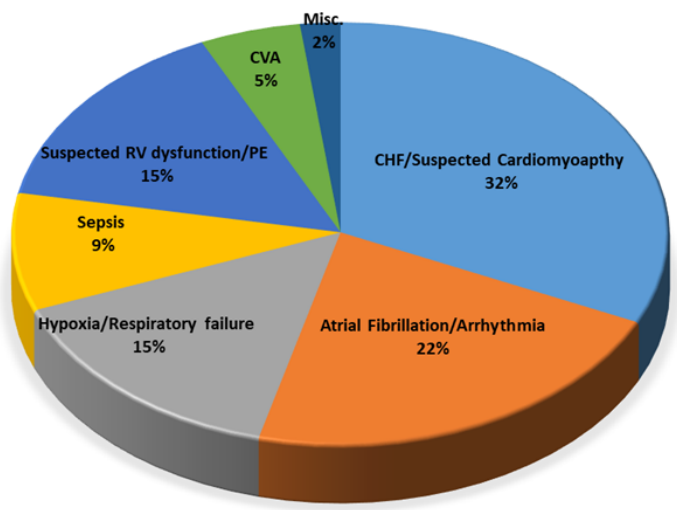

Figure 1c

DURATION

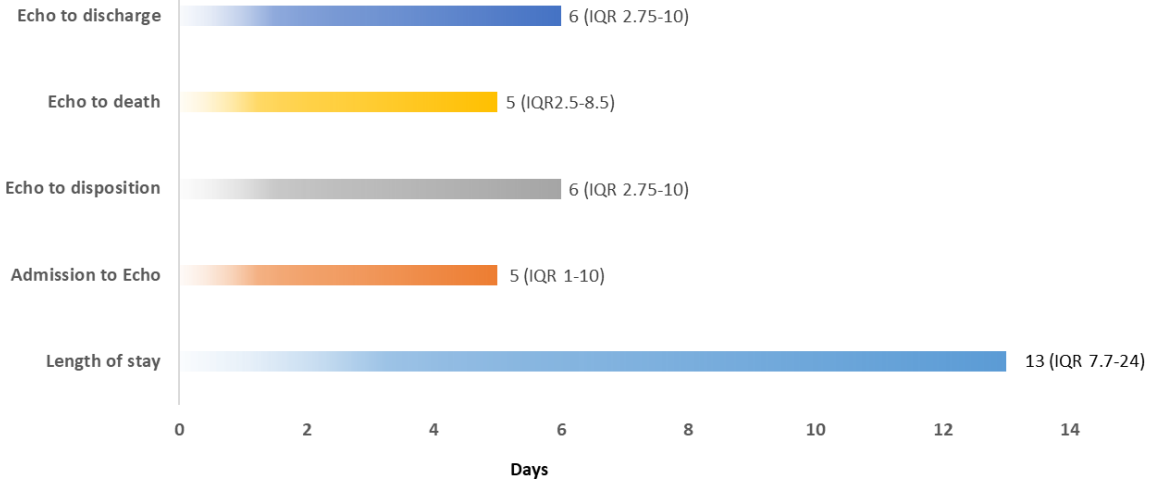

Figure 2 a- Impact of age on survival 


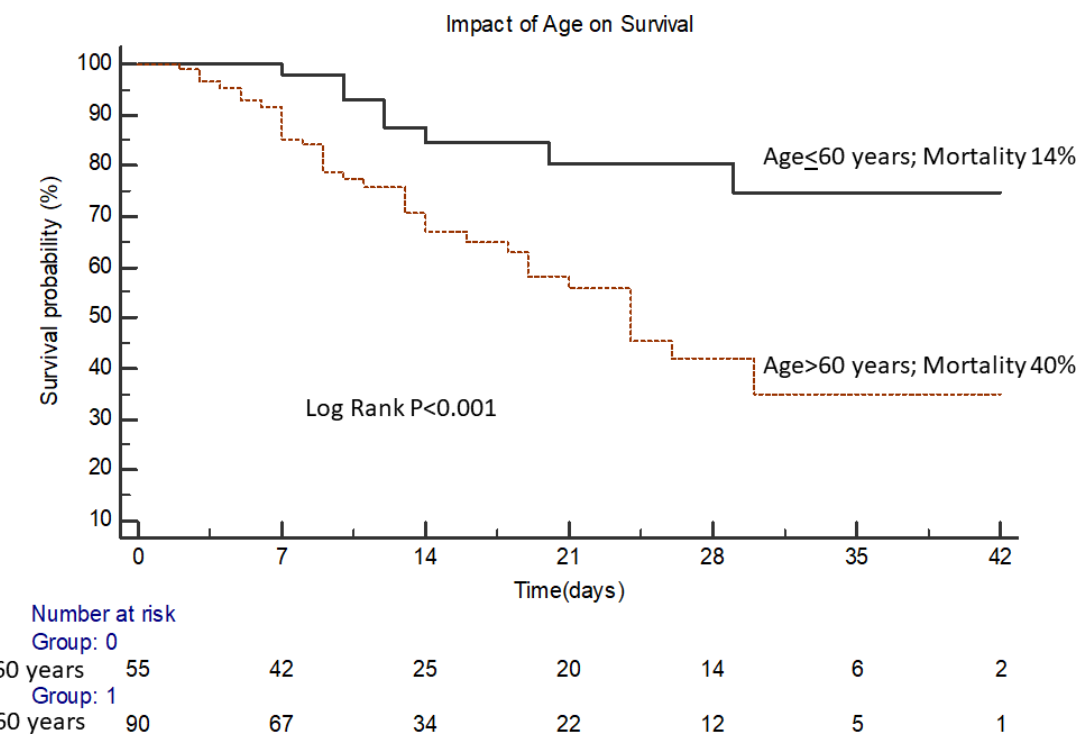

Figure 2b- Impact of pre-existing CAD on survival

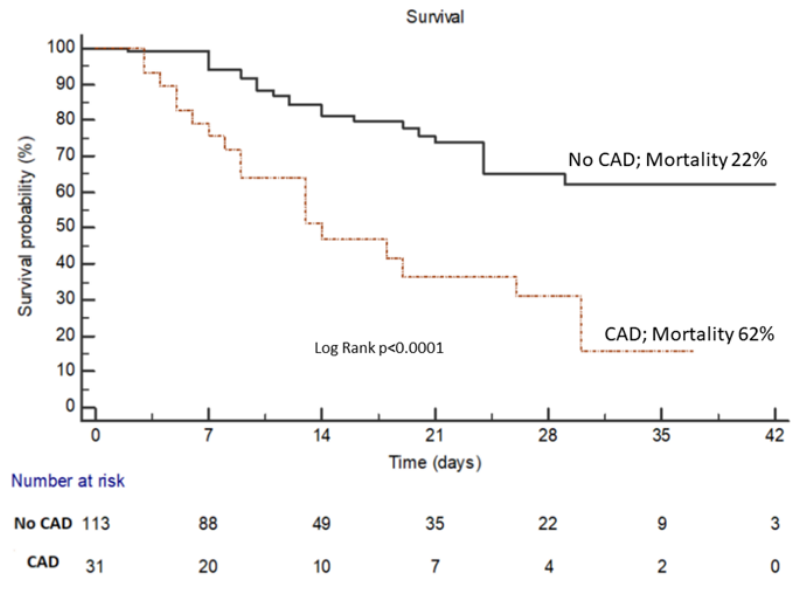

Figure 3a- Troponin level and outcome prediction 


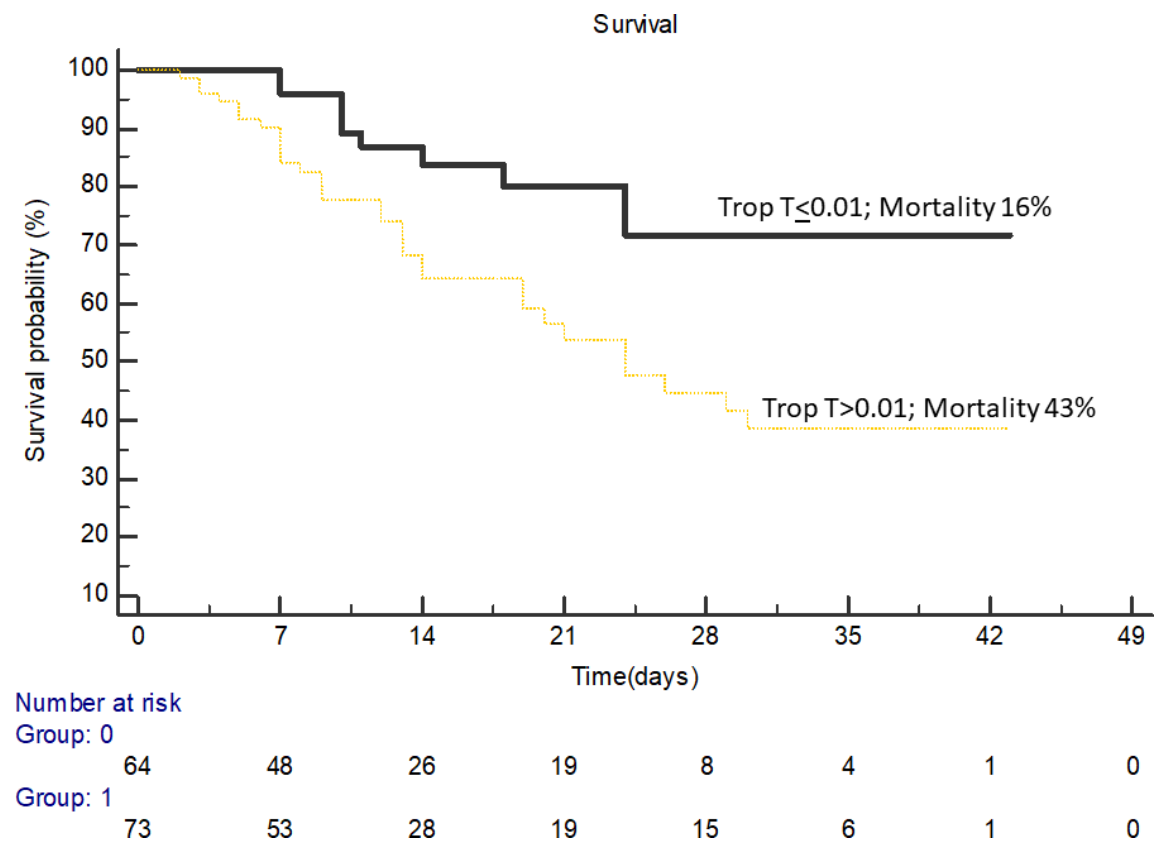

Figure 3b- Impact of left ventricular dysfunction on survival

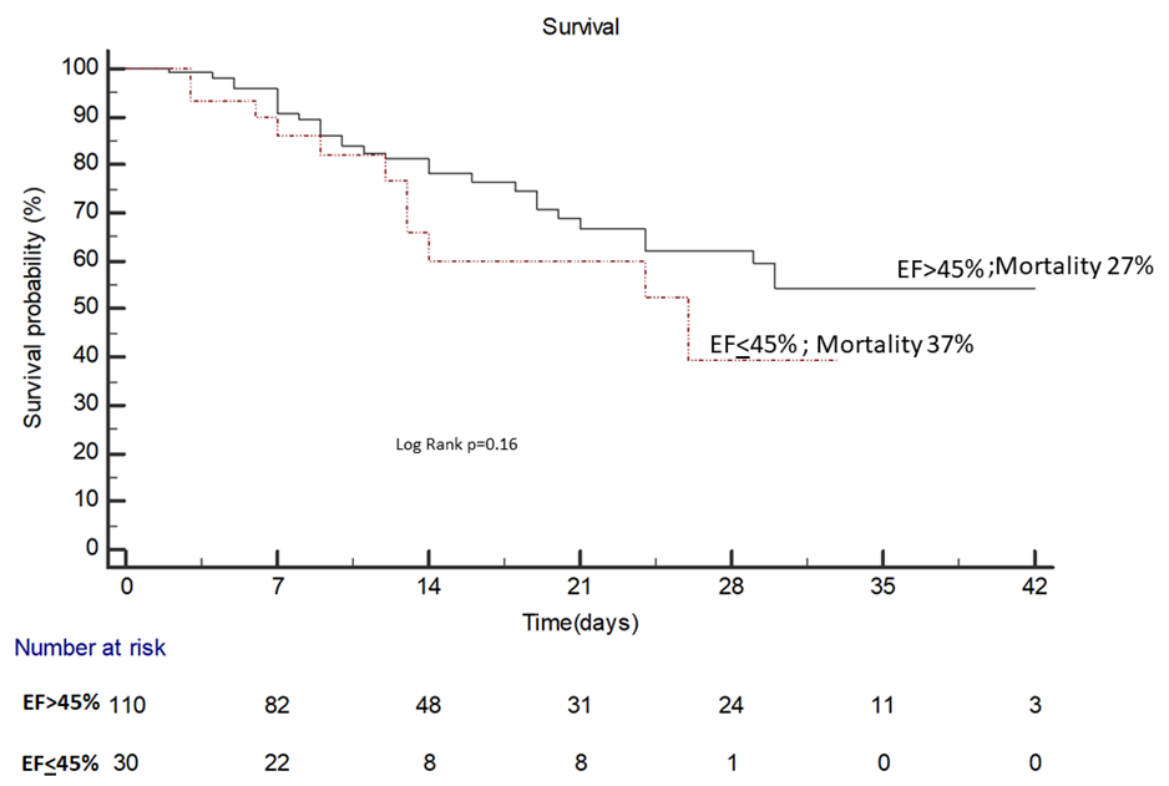

